Monatsschrift f. Geburtshülfe u. Gynäkologie 1927;75:I-IV

\title{
Contents, Vol. 75, 1927
}

\section{ïnhaltsverzeichnis.}

Seite

Zum Geleit 1

Wíssenschaftlîche Arbeîten und Veröffentlíchiingen. Von Paul

Straßmann 2

Mißlungene geburtshilflîche Eingriîfe. Von Geh.-Rat Prof. Dr.

Stoeckel-Beriin 7

Sterilität und Konstïtution. Von Prof. Dr. A. Mayer-Tübingen . . 21 Die Gelenkerkrankuлgen

während der Klimax. Von Geh.-Rat Prof.

W. $\mathrm{j}^{7} / 8 \mathrm{~s} \cdot$ Berlin . $\quad 26$

Über familiäres Auftrete $\pi$ von angeborenen Mißbildungen. Von

Geh.-Rat Prof. Dr. Otto Hildebrand-Beńin 32

Metrorrhagîen aus Thrombopenie und îhre Behandlung. Von Geh.-

Rat Prof. Dr. Georg Klemperer-Btím

35

Zur Beurteilung der Keimdrüsenpräparate. Von Geh.-Rat Prof. Dr.

C. Posner-Berlin

42

Der gegenwärtíge Stand der Lungenprobe. Von Geh.-Rat Prof. Dr.

F. Straßmann-Berl'm 47

Pertubation und Salpingostomîe zur Beseitigung der Sterilität. Von

Dr. H. Fuchs-Oanzig 56

Zur Frage des Hallux valgus. Von Prof. Dr. Richard Mühsam-Beńin 65

Die Operation des Prolapses und der Retroflexio uteri fixata. Von

Dr. Gotthard Schubert-Beuiben

Die Bedeutung der Zystoradiographie îür die Diagnostik der Harnröhren- und Blasenfisteln. Von San.-Rat Dr. Arthur Lewin-

Berlin 76

Brauchbare und unbrauchbare Abtreibungsmittel. Von Med.-Rat Dr.

Georg Straßmann-Bresia,u 79

Primäres Ureterkarzinom. Von Dr. Hans L. Posner-jüterbog . 86

Zur Frage über die Nierenausschaltung mittels Ureterknotung nach

Ureterresektion. Von Prof. Dr./. Braude-1·Aoskau .... 90

Experimentelle Untersuchungen zur Frage über die Ureterknotung.

Von Dr. E. M. Schwarsmann-Moskm

103

Pyelitis gravidarum. Von Dr. Paul Meyer-BtxWn 109

Eine angeborene kystomartige Bildung des rechten Ovarium. Von

Dr. Hans-Otto Neumann-Marburg 123

Inhaltsverzeichnis.

Originalarbeiten. Seít $\theta$

Benthin, W., Zur Prolapstherapie 384 
Bratide, J., Zur Frage über die Nierenausschaltung mittels Ureter-

knotung nach Ureterresektion.

90

Braun, Paul, Zur Technik der vaginalen Inzision 261

Brock, James, Gedanken über Nachgeburtsblutungen 250

Dörff'el, J., Über beginnende Blasenmolenbildung bei verhaltenem

(missed) Abort 391

Fink, Karl, Spasmen im Durchtrittssclilauch intra partum .... 365 Friedmann, Ernst, Die

Lumbalanästhesie bei Sectio caesarea $\cdot 163$ Fuchs, H., Pertubation und Salpingostomie zur

Beseitigung der

Sterilität 56

Gammeltoft, S. A., Die kongenitale Lues und ihre Bedeutung für

das Kind $\quad 400$

Heinrichsbauer, Franz, Uber angeborene Hautdefekte beim

Neugeborenen mit besonderer Berücksichtigung ihrer Atiologie 267 Hildebrand, Otto, Über

familiäres Auí'treten von angeborenen

Mißbildungen 32

His, W., Die Gelenkerkrankungen während der Klimax 26

Högler, Hans, Ursache und Behandlung der Geburt in Vorder-

hauptslage $\quad 349$

Joachimovits, Robert, Beitrag zur Klinik des Hydrops tubae

profluens $\quad 520$

Juhl, A. siehe Runge, $\mathrm{H}$.

Jung, Martin, O'ber den Begriff des Uterus pseudounicornis . . 131

Kamniker, Hellmut, Ursachen und Behandlungsergebnisse der

Q,uerlagen 233

Klaí'ten, E., Diabetes insipidus und Schwangerschaft

Klemperer, Georg, Metrorrhagien aus Thrombopenie und ihre

Behandlung 35

Küstner, Heinz, Hypoplasie der weiblichen Genitalien durch

Unterernährung in den Entwicklungsjahren 257

Zunahme geburtshilflicher Komplikationen in den letzten Jahren 524

Lahm, W., Zur Diagnose und Genese eines doppelseitigen soliden

und teilweise adenomatösen Ovarialtumors (Adenofibrosis). Zu-

gleich ein Beitrag zurn Adenoma endometroides 508

Lewin, Arthur, Die Bedeutung der Zystoradiographie für die

Diagnostik der Harnröhren- und Biasenfisteln 76

Martin, A., Schlußwort zur Straßmann-Festschrift 187

Mayer, A., Sterilität und Konstitution. . 21

Meyer, Paul, Zum Geleit der Straßmann-Festschrift 1

Pyelitis gravidarum 109

Mühsam, Richard, Zur Frage des Hallux valgus 65

Neumann, Hans-Otto, Eine angeborene kystomartige Bildung

des rechten Ovarium 123

Otto, G. E., Die Naegele-Zange am Steiß 143

Posner, C, Zur Beurteilung der Keimdrüsenpräparate

- L., Primäres Ureterkarzinom 86 
Rosenblatt, J., Salpingographie bei wegen Sterilität nach

Alexander-Adams operierten Frauen (vorläufige Mitteilung). . 382

$\uparrow Y \quad$ Inhaltsverzeichnis.

Seite

Eunge, H. uhd A. Juhl, Aminosäurebestimmungen nach Herzfeld

bei Schwangeren und Eklamptischen 463

Schubert, Gotthard, Die Operation des Prolapses und der Retro-

flexio uteri fixata 69

Schwarzmann, E. M., Experimentelle Untersuchungen zur Frage

über die Ureterknotung 103

Seitz, Ludwig, Die SchwangerschaftsveränderungenimLichte der

modernen Forschung und in ihrem Zusammenhang mit den

Schwangerschaftstoxikosen. I323

Die Schwangerschaftstoxikosen (Gestosen) und Schwanger-

schaftsdyskrasien im Lichte moderner Forschung. II 469

Sellheim, Hugo, „Schwebende Pein”, ein typisches gynäkolo-

gisches Krankheitsbild 368

Stahl, Albert, Über einen Fall von kombinierter Hemmungs-

mißbildung 150

Stoeckel, Walter, Mißlungene geburtshilfliche Eingriffe .... 7

Straßmann, Erwin, Die Ursache der Arkadie 172

F., Der gegenwärtige Stand der Lungenprobe

Georg, Brauchbare und unbrauchbare Abtreibungsmittel .... 79 - Paul, Wissenschaftliche

Arbeiten und Veröffentlichungen ... 2

Vogt, E., Praktische Erfahrungen mit der Bluttransfusion 262

Zimmer, Gerhard, Über Röntgenbestrahl·ung bei Myomen. . . . 157

Zinsstag, G., Schwangerschaft und Mitralstenose 498

Fraenkel, L., Eine Studienfahrt in den Vereinigten Staaten . . . 530

Sammelbericht.

Die gynäkologische Strahlentherapie im Jahre 1925. Von Dr. C. H.

Engelbrecht-Erlangen 189

Dammschutz, Dammriß und Dammnaht. Von Ed. Martin-Elberfeld 414

Vereíns- und Literaturbeilage.

Original-Sitzungsberichte aus geburtshilflich-gynäkologischen Gesellschafte $\pi$ :

Naturforscherversammlung in Düsseldorf. 19.-24. IX. 1926.

Stimmungsbericht von Hugo Sellheim 226

Gemeinschaftliche Sitzung mit der Deutschen Gesellschaft für

Kinderheilkunde. Sonntag, den 19. September 1926283

Geburtshilflich-gynäkologische Sektion 288

Gesellschaft für Geburtshilfe und Gynäkologie in Berlin.

Sitzungen vom 25. VI. und 9. VII. 1926422

Südostdeutsche Gesellschaft für Geburtshilfe und Gjmäkologie.

I. Tagung vom 2. V. 1926 in Breslau 432

Gjaiäkologische Gesellschaft in Breslau.

Sitzung vom 15. VI. 1926209

Sitzung vom 26. X. $1926 \quad 539$ 
Einladung zur XX. Tagung der „Deutschen Gesellschaft für Gynä

kologie" vom 8.-11. VI. 1927 in Bonn 460

Buchbesprechügen 227, 451, 546

Liíeraturverzeïch $\pi$ is $\quad 228,317,455,552$

Personalien und Tagesnachrichten 462,558

Druckfehlerberíchtígung 227

Sachregister für Band LXXI-LXXV . 559

Namenregister für Band LXXI-LXXV 571 Arbeiten aus der Straßmannschen KПniki seite

Über den Begriff des Uterus pseudounicornis. Von Dr. Martin

Jung-Y!tïWiii 131

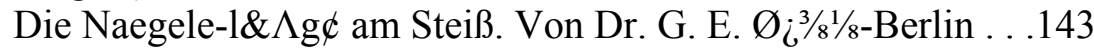

Uber einen Fall von kombinierter Hemmungsmißbildung. Von

Dr. Albert Sfø $\varnothing^{7} / 8 /$-Berlin 150

Uber Ròntgenbestrahlung bei Myomen. Von Dr. Gerhard Zimmer-

Berlin 157

Die Lumbalanästhesie bei Sectio caesarea. Von Dr. Ernst Friedmann-BerYm 163

Die Ursache der Arkadie. Von Or.Erwin Straßmann-BzrYm . .172

Schlußwort. Von Geh.-Rat Prof. Dr. A. Martin 187

Die gynäkologische Strahlentherapie im Jahre 1925. Sammelbericht von Dr. C. H. Engelbrecht-EvL·ngeh ... 189

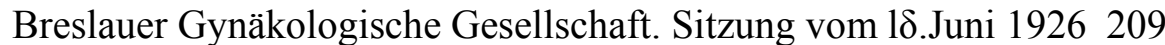

Buchanzeigen 227

Literatur-Verzeichnís 229 